\title{
Necesidades familiares y educativas de alumnos durante el confinamiento por COVID-19.
}

\section{Family and educational needs of students during COVID-19 confinement.}

Fecha de recepción: 26-05-2021

Fecha de aceptación: 01-12-2021
Juan Núñez-Sánchez

Universidad de València.

Marcela Núñez-Sánchez

Equipo Psicopedagógico Segorbe

Ana Soto-Rubio

Universitat de València.

\section{resumen/alsstract:}

El presente estudio ha tenido por objetivo estudiar el impacto psicológico del confinamiento domiciliar por la COVID-19 en escolares españoles y sus familias. Participaron 90 progenitores, siendo el 90\% madres. En relación a los hijos, el 84\% acudía a educación primaria. Se evaluaron variables sociodemográficas y psicopedagógicas, así como satisfacción con la vida, inteligencia emocional, estrategias de afrontamiento, estado emocional y datos relacionados con la COVID-19 (impacto, información, recursos y medidas tomadas). Se llevó a cabo un estudio transversal mediante encuesta online en coordinación con los departamentos de orientación de los colegios participantes. Se realizaron análisis descriptivos y de comparación de medias. Los resultados indican una percepción positiva de los padres respecto al rendimiento académico de sus hijos durante el confinamiento, pero negativa en lo referente a las medidas tomadas en relación a la COVID-19 por parte de las entidades responsables. Los progenitores también perciben como insuficientes los recursos y la información relativos a la COVID-19 proporcionados por dichas entidades durante el confinamiento. Se observa un mayor impacto psicológico en las familias de los escolares más jóvenes, y un menor impacto psicológico de las familias cuyos hijos reciben sesiones terapéuticas de audición y lenguaje.

The aim of the present study was to study the psychological impact of home confinement by COVID-19 on Spanish schoolchildren and their families. Ninety parents participated, 90\% of whom were mothers. Of the children, 84\% attended primary school. Sociodemographic and psycho-pedagogical variables were assessed, as well as life satisfaction, emotional intelligence, coping strategies, emotional state and data related to COVID-19 (impact, information, resources and measures taken). A cross-sectional study was carried out though an online survey in coordination with the guidance departments of the participating schools. Descriptive and mean comparison analyses were performed. The results indicate a positive perception of parents regarding the academic performance of their children during confinement, but negative regarding the measures taken in relation to COVID-19 by the responsible entities. Parents also perceive as insufficient the resources and information related to COVID-19 provided by these entities during the confinement. A greater psychological impact is observed in the families of younger schoolchildren, and a lesser psychological impact in families whose children receive hearing and language therapy sessions.

\section{palabras clave/keywords:}

COVID-19, necesidades educativas especiales, necesidades familiares, escolares, bienestar familiar.

COVID-19, special educational needs, family needs, school children, family well-being. 


\section{Introducción}

En la actualidad, la sociedad se enfrenta a la pandemia por COVID-19 y las numerosas consecuencias que ésta conlleva no sólo en el ámbito sanitario sino también en las esferas de lo económico y lo social, tanto a nivel familiar como individual (Duan y Zhu, 2020). El impacto psicológico de esta situación de pandemia comienza también a hacerse patente, no sólo a nivel individual con el surgimiento o agravamiento de psicopatologías, sino también alterando las dinámicas de convivencia y funcionamiento familiar (Brooks et al., 2020) Dicha alteración familiar incluye lidiar con dificultades no sólo directamente relacionadas con la COVID-19, sino también con las medidas tomadas para evitar la propagación de la misma. En España, como en la gran mayoría de los países afectados, una de estas medidas ha sido el confinamiento domiciliario y la suspensión de las actividades escolares en momentos puntuales del desarrollo de la pandemia en que se consideraba necesario. Tal es el caso del confinamiento domiciliario que tuvo lugar entre los meses de marzo y mayo de 2020, momento en el que se centran los datos del presente estudio (Sandín et al., 2020).

Aunque existen estudios previos sobre los efectos del confinamiento en el estado psicológico de los ciudadanos en otras situaciones de riesgo epidémico o vírico (Brooks et al., 2020), los estudios específicos sobre el confinamiento por COVID-19 son todavía escasos, lo que justifica estudios como el presente. La situación de pandemia y el confinamiento domiciliario suponen una fuente de estrés importante, así como un alto riesgo de malestar y alteración psicológica, requiriendo estrategias de afrontamiento específicas (Valero et al., 2020). Esta situación pone de manifiesto la importancia de generar estrategias funcionales y concretas para la situación actual; y cuidar la salud mental de toda la población (Jiloha, 2020) Por otra parte, varios autores justifican la necesidad de intervenir psicológicamente desde diferentes niveles y ámbitos y no limitar el apoyo al área sanitaria, actuando no sólo cuando ya se observan dificultades sino también de manera preventiva a través del apoyo psicológico y la facilitación y fortalecimiento de estrategias y recursos (Jiloha, 2020; Marques et al., 2020).

En cuanto a los factores relacionados con el impacto del confinamiento en las familias, estudios como el de Brooks y colaboradores señala que dicho impacto estará relacionado con la duración, siendo mayor cuanto más largo sea el confinamiento (Brooks et al., 2020). Otro de los factores a tener en cuenta sería el nivel de vulnerabilidad de algunos colectivos (WHO, 2020; Jiloha, 2020). Entre los grupos más vulnerables en situaciones como las del confinamiento por pandemia destaca la población infantil, que requiere una atención específica para facilitar su adaptación y promover un desarrollo saludable sin secuelas psicológicas (Cid et al., 2020). Aunque la población infantil no parece desarrollar, en la mayoría de los casos, síntomas graves de COVID-19 (Swann et al., 2020), su estado psicosocial y físico se ve afectado por el confinamiento, existiendo un mayor riesgo de psicopatología (Valero et al., 2020). Los cambios significativos en los hábitos y rutinas de los niños pueden repercutir negativamente en su salud mental (Paricio y Pando, 2020) originando alteraciones en el patrón de sueño, alimentación y conducta en general (Valero et al., 2020) así como problemas en el aprendizaje (Pérez, 2020), pudiendo llegar incluso a sufrir depresión, fobias, y estigmatización (Tran et al., 2020). Estas alteraciones se pueden clasificar en conductas internalizantes, de contenido principalmente emocional; y 
externalizantes, caracterizados por la expresión de conflictos (Garaigordobil y Maganto, 2012). En las conductas internalizantes predomina el retraimiento en los conflictos, por lo que se termina por perturbar el pensamiento, el desarrollo y la autonomía del menor; mientras que en las conductas externalizantes destacan la agresión, la actuación y la impulsividad; resultando en un rendimiento académico deficitario (Garaigordobil y Maganto, 2014).

Finalmente, los padres y madres también son vulnerables al desgaste psicológico que supone el confinamiento y las dificultades y retos que éste supone. Entre estas dificultades cabe destacar la adaptación a un sistema de educación telemático; la adaptación propia del empleo al contexto domiciliario y las dificultades de conciliación que esto supone; la numerosas preocupaciones generadas directamente por la emergencia sanitaria, como miedo al contagio, cuidado de un familiar contagiado, entre otras; la preocupaciones de índole económico, y aquellas relacionadas con el desempeño académico y el bienestar psicológico de sus hijos (Espino-Díaz et al., 2020) Aunque algunos padres y madres serán capaces de afrontar de forma resiliente la situación de pandemia, para una parte muy importante ésta potenciará las vulnerabilidades ya existentes o hará aparecer nuevos problemas, especialmente relacionados con la ansiedad y el estrés (Horesh y Brown, 2020).

Por todo ello, es necesario estudiar el estado psicológico de los padres y madres y el posible impacto que el confinamiento y la COVID-19 ha tenido en ellos, no sólo prestando atención a las posibles variables de riesgo y malestar, sino también a las de protección y bienestar (Blasco-Belled et al., 2020). Todo ello con el fin no sólo de brindarles la atención que merecen y velar por su salud; sino también para promover y velar por el bienestar de la familia en su conjunto, ya que el estado mental de los padres y madres se ha visto relacionado con el desarrollo personal y escolar de sus hijos (Lau et al., 2018; Lui et al., 2020; McLaughlin et al., 2012; Reupert y Maybery, 2016).

\section{Material y métodos}

\section{Participantes}

En el estudio participaron 90 progenitores de niños en edad escolar, de los cuales el $90 \%$ eran madres y el $8,9 \%$ padres, con una edad media de 41,91 años $(\mathrm{DT}=4.974)$ y un rango de 28 a 55 años. El 52,2\% de los participantes tienen a sus hijos escolarizados en centros públicos y el $47,8 \%$ en centros privados o concertados. El $84 \%$ de los escolares están en educación primaria, el $8 \%$ en educación secundaria y el $6 \%$ en educación infantil.

En cuanto al sexo de los escolares, el $44 \%$ son niñas y el $46 \%$ niños.

\section{Instrumentos}

Se han evaluado las características sociodemográficas de los padres y madres y de sus hijos mediante un cuestionario ad hoc, así como las variables educativas y psicológicas: edad, sexo, nivel de estudios, diagnóstico, asistencia a pedagogía terapéutica y/o audición y lenguaje.

Para evaluar la satisfacción psicológica, se ha utilizado el cuestionario SWLS (Satisfaction With Life Scale) de Diener et al. (1985), adaptación española de Atienza, Pons, Balaguer y García-Merita (2000). Consta de cinco ítems en escala Likert con valores de 1 a 5 donde 1 
es "totalmente en desacuerdo" y 5 es "totalmente de acuerdo" a través de los que evaluar el nivel de percepción de satisfacción global con la vida. Posee una consistencia interna de 84 y resultados positivos en el Análisis Factorial Confirmatorio (Atienza et al., 2000)

La inteligencia emocional se ha evaluado mediante el cuestionario TMMS-24 (Trait MetaMood Scale), de Salovey, Mayer, Goldman y Turvey (1995); adaptado al español por Fernández-Berrocal, Extremera y Ramos (2004). Está compuesto por 24 ítems con opción de respuesta tipo Likert con 5 opciones de respuesta, donde el entrevistado debe evaluar desde (1) "nada de acuerdo" hasta 5 "totalmente de acuerdo" en cuanto a su inteligencia emocional intrapersonal. Posee una consistencia interna de entre 0.85 y 0.88 mediante el alfa de Cronbach (Lebret, 2018)

Las estrategias de afrontamiento se han evaluado mediante el COPE-28 (Inventario Breve de Afrontamiento), la versión española del BRIEF-COPE de Carver (1997); adaptado por Crespo y Cruzado (1997) El COPE-28 proporciona información sobre trece tipos de respuesta al estrés. Consta de 28 ítems que se plantean en función de la acción y respuesta que las personas dan en una escala ordinal con cuatro alternativas (de 0 a 3 ), entre "nunca hago esto" y "siempre hago esto". Posee una consistencia interna de 78 (Mate et al., 2016) El estado emocional se ha evaluado mediante la escala SPANE (Escala de Experiencias Positivas y Negativas), de Diener et al.(2009); adaptada al español por Carmona y VillegasRobertson (2018). Consta de 12 ítems tipo Likert, con puntuaciones comprendidas entre 1 "nunca" y 5 "siempre"; que proporcionan información sobre la experiencia emocional positiva y negativa; así como del equilibrio emocional. En relación a las propiedades psicométricas, el alfa de Cronbach para la dimensión positiva es de .92, mientras que para la dimensión negativa es de .83 (Carmona-Halty y Villegas-Robertson, 2018).

En referencia al impacto que el confinamiento ha tenido en las familias, se han diseñado ítems que valoran aspectos de la conducta en el niño, tanto externalizantes (problemas de sueño, alimentación, rabietas, desobediencia), como internalizantes (alegría, tristeza, nerviosismo, aburrimiento) y el nivel de estrés que los participantes manifiestan relacionados con dificultades en la comunicación y en la realización de los deberes escolares durante el confinamiento.

\section{Procedimiento y análisis de datos}

La evaluación se ha realizado en formato de encuesta online debido a las recomendaciones sanitarias sobre el contacto social, cuya cumplimentación se llevaba a cabo en 20-30 minutos. A través de los departamentos de orientación de los colegios, se puso a disposición de los padres y madres que voluntariamente quisieran participar. Los padres y madres fueron informados del objetivo del estudio y de cómo se garantizaba su anonimato y confidencialidad de acuerdo a los principios de la Declaración de Helsinki y contando con la aprobación del comité de ética de la Universitat de València. Los datos fueron obtenidos durante el mes de junio del 2020. Se ha utilizado el paquete estadístico SPSS 25 (Statistical Package for the Social Science) IBM Corporation, para realizar los análisis descriptivos de las variables sociodemográficas y psicopedagógicas, así como comparaciones de medias. 


\section{Resultados}

\section{Perfil Sociodemográfico y psicopedagógico}

El perfil de los padres y madres del estudio está formado mayoritariamente por mujeres $(81 \%)$, con estudios universitarios $(53,3 \%)$ o de secundaria $(34,4 \%)$, que envían a sus hijos de forma similar a colegios públicos $(52,2 \%)$ o privados/concertados $(47,8 \%)$. En la mayoría de los casos tienen a sus hijos en educación primaria $(84,4 \%)$, siendo aproximadamente la mitad de ellos niños $(51,1 \%)$ y la otra mitad niñas $(48,9 \%)$.

Tabla 1.- Estadísticos descriptivos de la muestra

\begin{tabular}{|c|c|c|c|}
\hline & & Frecuencia & Porcentaje \\
\hline \multirow{2}{*}{$\begin{array}{l}\text { Sexo del tutor } \\
\text { encuestado }\end{array}$} & Femenino & 81 & 90,0 \\
\hline & Masculino & 9 & 10,0 \\
\hline \multirow[t]{4}{*}{ Nivel de formación } & Sin estudios & 1 & 1,1 \\
\hline & Estudios primarios o equivalentes & 10 & 11,1 \\
\hline & Estudios secundarios o equivalentes & 31 & 34,3 \\
\hline & Estudios universitarios o equivalentes & 48 & 53,3 \\
\hline \multirow{2}{*}{$\begin{array}{l}\text { Tipo de centro donde } \\
\text { cursa el menor }\end{array}$} & Público & 47 & 52,2 \\
\hline & Privado/concertado & 43 & 47,8 \\
\hline \multirow{3}{*}{$\begin{array}{l}\text { Etapa educativa que } \\
\text { cursa }\end{array}$} & Infantil & 6 & 6,7 \\
\hline & Primaria & 76 & 84,4 \\
\hline & Secundaria & 8 & 8,9 \\
\hline \multirow{2}{*}{$\begin{array}{l}\text { Género del hijo más } \\
\text { pequeño en primaria }\end{array}$} & Femenino & 44 & 48,9 \\
\hline & Masculino & 46 & 51,1 \\
\hline \multirow{5}{*}{ Diagnósticos } & Altas capacidades intelectuales & 3 & 3,3 \\
\hline & TDAH & 8 & 8,9 \\
\hline & DEA & 11 & 12,2 \\
\hline & Comunicación & 4 & 4,4 \\
\hline & Diversidad sensorial & 1 & 1,1 \\
\hline \multirow{2}{*}{$\begin{array}{l}\text { Servicios } \\
\text { psicopedagógicos }\end{array}$} & PT & 18 & 20 \\
\hline & AyL & 9 & 10 \\
\hline \multirow[t]{4}{*}{ Calificaciones } & Son algo peores & 9 & 8,9 \\
\hline & No han cambiado & 59 & 65,6 \\
\hline & Son algo mejores & 19 & 21,1 \\
\hline & Son mucho mejores & 4 & 4,4 \\
\hline
\end{tabular}

Nota: TDAH = Trastorno por Déficit de Atención e Hiperactividad; DEA: Dificultad Específica del Aprendizaje; PT: Pedagogía Terapéutica; AyL: Audición y Lenguaje. 
Los resultados descriptivos indican una presencia de alumnos con discapacidad en la muestra del 6,7\%, presentando un 12,2\% una dificultad específica de aprendizaje, y el 8,9\% un trastorno por déficit de atención (con o sin hiperactividad).

Sobre los servicios especializados de los que disponen los alumnos previamente al confinamiento, el 20\% indicó que recibían pedagogía terapéutica, y el 10\% audición y lenguaje. Por último, la percepción de los padres y madres sobre el rendimiento académico durante el confinamiento es mayoritariamente positiva, ya que el $65 \%$ de los padres y madres indica que no ha notado una variación en las notas y el 21,1\% indica que son algo mejores que antes. Los detalles de los datos sociodemográficos pueden verse en la Tabla 1.

\section{Impacto y bienestar psicológico por COVID-19}

En base a los resultados obtenidos no se observan cambios en alteraciones internalizantes ni externalizantes en los menores con puntuaciones de $\mathrm{M}=3.13$ (DT = .334) y $\mathrm{M}=3.16$ (DT = .396), siendo 1 mínimo impacto y 5 máximo impacto. Por otra parte, sí se observan alteraciones en el caso de los padres en la variable impacto psicológico asociada a la COVID-19 ( $\mathrm{M}=4.01$, DT =.753). Así mismo, en lo que refiere al bienestar psicológico de los padres, considerando el componente afectivo o emocional, los resultados sugieren una mayor presencia de emociones positivas $(\mathrm{M}=21.62$ y DT $=3.30)$ frente a emociones negativas $(\mathrm{M}=15.46$ y DT $=4.01)$, lo que implica un balance hedónico positivo. Por su parte, en lo que refiere al componente cognitivo, se observan niveles medios, con valores de $3,70(\mathrm{DT}=.665)$ sobre cinco en la escala de satsifacción con la vida. Los resultados pueden observarse en la Tabla 2.

Tabla 2.- Impacto y bienestar psicológico por COVID

\begin{tabular}{llll}
\hline & M (DT) & Mínimo & Máximo \\
\hline Impacto hijos & $3,1404(.3348)$ & 2.11 & 4 \\
Impacto padres & $4,0139(.75361)$ & 2 & 5 \\
Alteración externalizante hijo & $3,1690(.3963)$ & 1.86 & 4,14 \\
Alteración internalizante hijo & $3,1317(.4668)$ & 1.57 & 4,29 \\
Spane positivo & $21,62(3.30)$ & 15 & 30 \\
Spane negativo & $15,46(4.01)$ & 6 & 27 \\
Equilibrio afectivo & $6,16(6.50)$ & -8 & 24 \\
SWLS & $3,70(.665)$ & 1,80 & 5 \\
\hline
\end{tabular}

Nota: M = Media , DT: Desviación Típica, SWLS: Escala de satisfacción con la vida

\section{Percepción de los padres y madres frente a la COVID-19}

En lo referente a la percepción que tenían los padres y madres frente a la COVID-19 se observa que la mayoría de las variables evaluadas son percibidas negativamente. En este sentido, se observan valores inferiores a 3 sobre cinco tanto en la satisfacción que manifiestan con los recursos que se han implantado desde el colegio $(\mathrm{M}=2.813$, $\mathrm{DT}=.938)$, como con 
la información que se les hace llegar desde los organismos oficiales $(\mathrm{M}=2.696, \mathrm{DT}=.839)$ así como con las medidas tomadas por las diferentes entidades responsables $(\mathrm{M}=2.685$, $\mathrm{DT}=.844)$. Así mismo, los datos señalan que los padres y madres consideran que ha habido un impacto significativo en su vida y en la de sus familias relacionado con la COVID-19 (M $=3.458, \mathrm{DT}=.858)$. Los resultados pueden observarse en la Tabla 3 .

Tabla 3.- Percepción de los padres y madres en relación a la COVID-19

\begin{tabular}{llll}
\hline & $\mathrm{M}(\mathrm{DT})$ & Mínimo & Máximo \\
\hline Recurso & $2,813(.938)$ & 1 & 5 \\
Información & $2,696(.839)$ & 1 & 5 \\
Medidas & $2,685(.844)$ & 1 & 5 \\
Impacto & $3,458(.858)$ & 1 & 5 \\
\hline
\end{tabular}

Nota: M = Media , DT: Desviación Típica

\section{Análisis de comparación de medias}

Se llevó a cabo un análisis de comparación de medias con respecto a las variables de estudio teniendo en cuenta la asistencia o no a pedagogía terapéutica (PT) y a audición y lenguaje (AyL) durante el confinamiento. Las diferencias significativas, así como el tamaño del efecto, se observan en la Tabla 4.

Tabla 4.- Comparación de medias en función de la asistencia o no a pedagogía terapéutica y a audición y lenguaje.

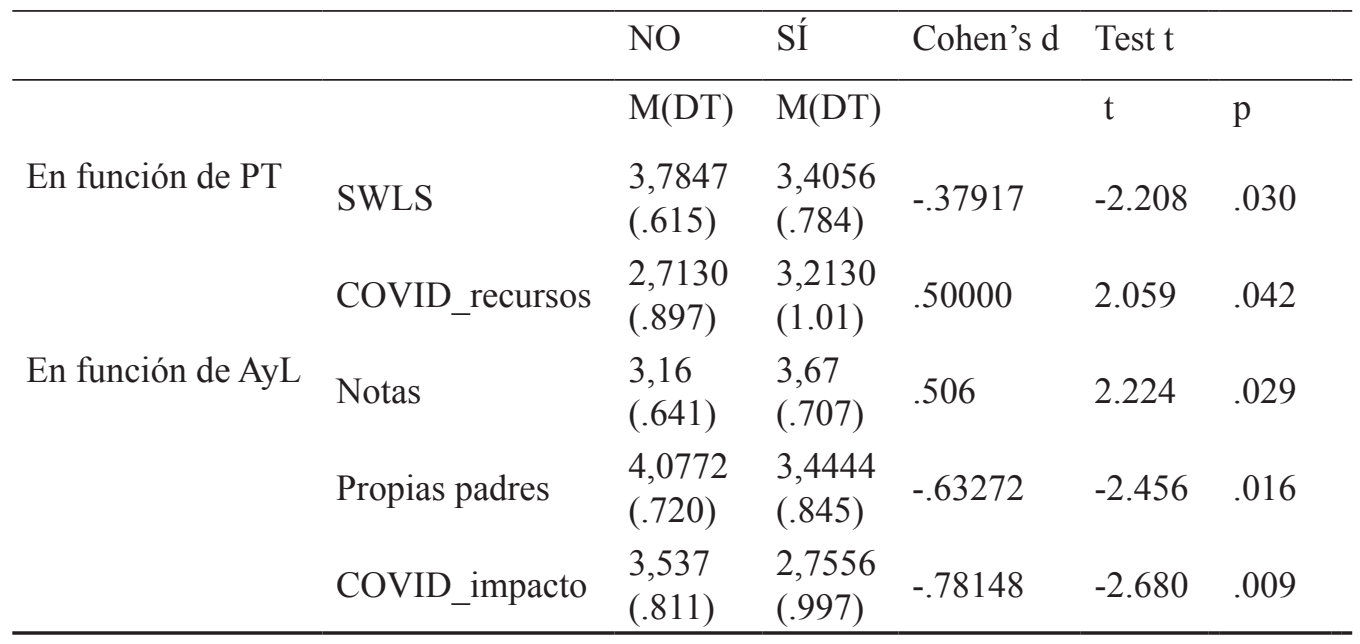

Nota: M= Media; DT= Desviación típica; PT= Pedagogía Terapéutica; AyL= Atención y

Lenguaje; SWLS= Escala de Satisfacción con la Vida; Cohen's d= tamaño del efecto; $\mathrm{p}=$ probabildiad. 
Los resultados del análisis de comparación de medias indican una mayor puntuación en la variable satisfacción con la vida en el grupo de padres y madres cuyos hijos no reciben sesiones de Pedagogía Terapeútica, así como una menor puntuación en los recursos facilitados por las entidades responsables para hacer frente a la COVID-19.

Finalmente, en lo referente a las sesiones de audición y lenguaje, los alumnos de este servicio han mejorado sus notas durante el confinamiento y sus padres y madres informan sobre un impacto a causa de la COVID-19, estrés por los deberes y comunicación con el centro, en comparación con los padres y madres de los niños que no asisten a este tipo de sesiones.

\section{Discusión}

El presente estudio ha tenido como objetivo general estudiar el impacto del confinamiento domiciliario a causa de la pandemia originada por la COVID-19 en las familias. En relación con las consecuencias educativas, a diferencia de la preocupación social generalizada (Tran et al., 2020), la percepción de la mayoría de los padres y madres es que sus hijos están obteniendo resultados académicos similares a los anteriores a la COVID-19, lo que puede deberse en parte a que la mayoría de la muestra ha mantenido la regularidad en sus clases a pesar del cambio de formato y a la juventud de los padres y madres, posiblemente familiarizados con los entornos virtuales.

Por el contrario, se observan consecuencias psicológicas en los niños, especialmente en conductas observables como el sueño, la alimentación o las conductas disruptivas, que en el estudio hemos evaluado bajo la categoría de conductas externalizantes. La alteración de los horarios y rutinas de los niños ha contribuido a una alteración de sus conductas cotidianas (Paricio del Castillo y Pando Velasco, 2020) y el malestar psicológico que han podido sufrir (Cid et al., 2020), lo que refuerza la importancia de proporcionar apoyo psicológico a los alumnos tras el encierro.

Asimismo, los padres y madres muestran un alto grado de desacuerdo o malestar con las medidas de información general, los recursos del centro y las medidas públicas relativas a la gestión de la COVID y la pandemia, lo que coincide con el malestar general evaluado en otros países (Johnson et al., 2020) y que podría aumentar la incertidumbre y la ansiedad experimentadas.

En cuanto a la alteración conductual observada en los niños y niñas durante el confinamiento, ésta aparece más acusada en relación a las conductas externalizantes frente a las internalizantes, lo que ya se ha observado en otros estudios al evaluar conductas como la alimentación, la actividad física o el sueño de los internos (E. Valero et al., 2020). A la hora de interpretar este resultado hay que tener en cuenta que esta alteración conductual tiene lugar durante el confinamiento, pero no por ello es debía a éste, sino que su origen puede estar en diferentes factores que ocurren durante el confinamiento y que pueden estar relacionados con la pandemia o no. No obstante, dado que se acusa la alteración conductual durante el confinamiento, es algo a tener presente de cara a futuras investigaciones, así como de cara al diseño de ayudas específicas para sobrellevar este tipo de situaciones cuidando el bienestar de los niños y niñas y el de sus familias. 
Por otra parte, en relación con las diferencias observadas según la asistencia o no a pedagogía terapéutica, los datos obtenidos al evaluar la satisfacción con la vida de los padres y madres indican una menor satisfacción en los padres y madres de los escolares que asisten a Pedagogía Terapéutica, un servicio de apoyo educativo para alumnos que presentan dificultades de aprendizaje. Este hecho podría explicarse por la afectación habitual que pueden presentar los padres de menores con dificultades del aprendizaje, asociada al estrés o sobrecarga de las necesidades de sus hijos (Auriemma et al., 2021; Zapata et al., 2013) así como por el empeoramiento en las dificultades del aprendizaje preexistentes a causa de la situación de confinamiento (Espino-Díaz et al., 2020).

Los resultados del presente estudio pueden guiarnos en situaciones venideras a la hora de tomar decisiones prácticas, teniendo en cuenta la demanda que hace la familia de información y recursos en situaciones de este tipo ya que en la actualidad sigue aplicándose confinamiento domiciliar de escolares en casos de contagio por COVID-19. Puede ser útil llevar a cabo asesoramiento familiar referente a la gestión del sueño, alimentación y conductas disruptivas durante confinamientos familiares e incorporar estas propuestas a las adaptaciones escolares en situaciones de confinamiento o similares.

Finalmente, en referencia a las diferencias observadas según la asistencia o no a sesiones de Audición y Lenguaje, un servicio dirigido a trabajar las habilidades académicas en menores con diagnósticos que afectan a su correcto uso de la comunicación, la escritura y la lectura, han supuesto, por un lado, mejores calificaciones en aquellos alumnos que sí las reciben, lo que puede deberse en parte al refuerzo instrumental en funciones lingüísticas transversales recibido alumnos, lo que puede deberse en parte al factor de apoyo que proporciona este servicio, así como a las instrucciones precisas sobre las dificultades de lectura y escritura que los especialistas pueden haber dado a los padres y madres durante el encierro, aliviando así su carga en términos de apoyo académico.

Limitaciones y futuras líneas de investigación

Aunque este estudio pretende aportar información relevante sobre la situación que viven los padres y madres de niños en edad escolar durante el encierro por COVID-19, no está exento de limitaciones. Por un lado, el número de participantes es limitado, lo que no permite un análisis más completo de los datos, pero las particularidades del inicio de la pandemia han dificultado el acceso a una muestra mayor. Al mismo tiempo, al ser este estudio transversal, no disponemos de datos sobre la evolución del impacto por confinamiento a lo largo del tiempo. Futuras investigaciones se beneficiarían de la inclusión de muestras más amplias, y de la medición de las variables en diferentes momentos para dilucidar mejor las relaciones entre ellas y la evolución de la situación de las familias a medida que avanzan la pandemia y el confinamiento.

A pesar de sus limitaciones, este estudio contribuye al conocimiento de la situación de los padres y madres de niños en edad escolar durante una situación tan particular y difícil como la del confinamiento en casa debido a la pandemia de COVID-19, especialmente en lo referente a algunas variables que pueden estar relacionadas con su bienestar y el de sus hijos. Los resultados del estudio aportan información novedosa y específica respecto al 
bienestar psicológico e impacto de alumnos que reciben atención especializada de pedagogía terapéutica y audición y lenguaje, presentándose éstos como recursos de protección no sólo para el alumno, si no para la familia ante posibles situaciones adversas como la que se vivió en España y en distintos países del mundo al confinar a la población para frenar la propagación del virus de la COVID-19.

Dado que desafortunadamente la situación de pandemia aún no ha terminado, la posibilidad de un nuevo confinamiento es real. Cualquier información que ayude a los padres y madres y a los niños en esta situación es relevante y necesaria.

\section{Agradecimientos}

A los padres y madres, niños y niñas, y al personal en los centros educativos, por su colaboración, tiempo y sinceridad en un momento tan difícil como es el estar confinados por una pandemia. Ana Soto-Rubio es beneficiaria de una beca posdoctoral financiada por el Fondo Social Europeo y la Generalitat Valenciana.

\section{Referencias}

Auriemma, D.L., Ding, Y., Zhang, C., Rabinowitz, M., Shen, Y. and Lantier-Galatas, K. (2021), Parenting Stress in Parents of Children with Learning Disabilities: Effects of Cognitions and Coping Styles. Learning Disabilities Research y Practice. Doi:10.1111/Idrp.12265

Angulo, R., y Albarracín, A. P. (2018). Validez y confiabilidad de la escala rasgo de metaconocimiento emocional (TMMS-24) en profesores universitarios. Revista Lebret, 10, 61-7.

Atienza, F. L., Pons, D., Balaguer, I., y García-Merita, M. (2000). Propiedades psicométricas de la Escala de Satisfacción con la Vida en adolescentes. Psicothema, 12(2), 314-319.

Blasco-Belled, A., Tejada-Gallardo, C., Torrelles-Nadal, C., y Alsinet, C. (2020). The costs of the COVID-19 on subjective well-being: An analysis of the outbreak in Spain. Sustainability, 12(15), 6243. https://doi.org/10.3390/ SU12156243

Brooks, S. K., Webster, R. K., Smith, L. E., Woodland, L., Wessely, S., Greenberg, N., y Rubin, G. J. (2020). The psychological impact of quarantine and how to reduce it: rapid review of the evidence. The Lancet, 395(10227), 912-920. https://doi.org/10.1016/S0140-6736(20)30460-8

Cabrera, L. (2020). Efectos del coronavirus en el sistema de enseñanza : aumenta la desigualdad de oportunidades educativas en España. Revista de Sociología de La Educación-RASE, 13(2, Especial, COVID-19), 114-139.

Carmona-Halty, M., y Villegas-Robertson, J. M. (2018). Escala de Experiencias Positivas y Negativas (SPANE): Adaptación y validación en un contexto escolar chileno. Interciencia, 43(May), 317-319. https://www.interciencia.net/wp-content/uploads/2018/05/317-CARMONA-43_5.pdf

Carver, C. S. (1997). You want to measure coping but your protocol's too long: consider the Brief-COPE. International Journal of Behavioral Medicine, 4, 92-100

Cid, M. F. B., Fernandes, A. D. S. A., Morato, G. G., y Minatel, M. M. (2020). Psychosocial care and the covid-19 pandemic: Reflections on care for children and adolescents living in socially vulnerable contexts. Multidisciplinary Journal of Educational Research, 10(2), 178-201. https://doi.org/10.447/remie.2020.5887

Crespo, M., y Cruzado, J. A. (1997). La evaluación del afrontamiento: adaptación Española del cuestionario COPE con una muestra de estudiantes universitarios. Análisis y Modificación de Conducta, 23, 797-830.

Duan, L., y Zhu, G. (2020). Psychological interventions for people affected by the COVID-19 epidemic. The Lancet Psychiatry, 7(4), 300-302. https://doi.org/10.1016/\$2215-0366(20)30073-0 
Diener, E., Wirtz, D., Tov, W., Kim-Prieto, C., Choi, D. W., Oishi, S., y Biswas-Diener, R. (2010). New well-being measures: short scales to assess flourishing and positive and negative feelings. Social Indicators Research, 97, 143-156.

Diener, E., Emmons, R. A., Larsen, R. J., y Griffin, S. (1985). The Satisfaction with Life Scale. Journal of Personality Assessment, 49, 71-75.

Espino-Díaz, L., Fernandez-Caminero, G., Hernandez-Lloret, C. M., Gonzalez-Gonzalez, H., y Alvarez-Castillo, J. L. (2020). Analyzing the impact of COVID-19 on education professionals. Toward a paradigm shift: ICT and neuroeducation as a binomial of action. Sustainability, 12(14), 5646. https://doi.org/10.3390/su12145646

Fernández-Berrocal, P., Extremera, N. y Ramos, N. (2004). Validity and reliability of the Spanish modified version of the Trait Meta-Mood Scale. Psychological Reports, 94, 751-755.

Fontanesi, L., Marchetti, D., Mazza, C., di Giandomenico, S. D., Roma, P., y Verrocchio, M. C. (2020). The effect of the COVID-19 lockdown on parents: A call to adopt urgent measures. Psychological Trauma: Theory, Research, Practice, and Policy, 12, 79-81. https://doi.org/10.1037/tra0000672

Garaigordobil, M., y Maganto, C. (2012). SPECI. Screening de problemas emocionales y de conducta. Madrid: TEA.

Garaigordobil, M., y Maganto, C. (2014). SPECI. Screening de problemas emocionales y de conducta infantil: Descripción y datos psicométricos. International Journal of Developmental and Educational Psychology, 4(1), 319-328. https://doi.org/10.17060/ijodaep.2014.n1.v4.618

Horesh, D., y Brown, A. D. (2020). Covid-19 response: Traumatic stress in the age of Covid-19: A call to close critical gaps and adapt to new realities. Psychological Trauma: Theory, Research, Practice, and Policy, 12(4), 331-335. https://doi.org/10.1037/TRA0000592

Isabel Mate, A., Manuel Andreu, J., y Elena Pena, M. (2016). Psychometric properties of the Spanish version of the Brief COPE Inventory (COPE-28) in a sample of teenagers. Behavioral Psychology-Psicologia Conductual, 24(2), 305-318.

Jiloha, R. (2020). View of COVID-19 and mental health. Advanced Research Publications, 5(1), 9-11. http://medical. advancedresearchpublications.com/index.php/Epidem/nternational/article/view/256/221

Johnson, M. C., Saletti-Cuesta, L., y Tumas, N. (2020). Emotions, concerns and reflections regarding the COVID-19 pandemic in argentina. Ciencia e Saude Coletiva, 25, 2447-2456. https://doi.org/10.1590/141381232020256.1.10472020

Lau, P., Hawes, D. J., Hunt, C., Frankland, A., Roberts, G., y Mitchell, P. B. (2018). Prevalence of psychopathology in bipolar high-risk offspring and siblings: a meta-analysis. European Child and Adolescent Psychiatry, 27(7), 823-837. https://doi.org/10.1007/s00787-017-1050-7

Lui, M., Lau, G. K., Tam, V. C., Chiu, H-M., Li, S. S., y Sin, K-F. (2020). Parents' Impact on Children's School Performance: Marital Satisfaction, Parental Involvement, and Mental Health. Journal of Child and Family Studies, 29(6), 1548- 1560. https://doi.org/10.1007/s10826-019-01655-7

Marques, E. S., de Moraes, C. L., Hasselmann, M. H., Deslandes, S. F., y Reichenheim, M. E. (2020). Violence against women, children, and adolescents during the COVID-19 pandemic: Overview, contributing factors, and mitigating measures. Cadernos de Saude Publica, 36(4). https://doi.org/10.1590/0102-311X00074420

McLaughlin, K. A., Gadermann, A. M., Hwang, I., Sampson, N. A., Al-Hamzawi, A., Andrade, L. H., Angermeyer, M. C., Benjet, C., Bromet, E. J., Bruffaerts, R., Caldas-de-Almeida, J. M., de Girolamo, G., de Graaf, R., Florescu, S., Gureje, O., Haro, J. M., Hinkov, H. R., Horiguchi, I., Hu, C., ... Kessler, R. C. (2012). Parent psychopathology and offspring mental disorders: Results from the WHO World Mental Health Surveys. British Journal of Psychiatry, 200(4), 290-299. https://doi.org/10.1192/bjp.bp.111.101253

Morales, F., Trianes, M., y Miranda, J. (2012). Diferencias por sexo y edad en afrontamiento infantil del estrés cotidiano. Electronic Journal of Research in Educational Psychology, 10(26), 95-110.

Paricio del Castillo, R., y Pando Velasco, M. F. (2020). Salud mental infanto-juvenil y pandemia de Covid-19 en España: cuestiones y retos. Revista de Psiquiatría Infanto-Juvenil, 37(2), 30-44. https://doi.org/10.31766/revpsij. v37n2a4

Pérez, R. (2020). Estudio de la influencia del confinamiento debido a la COVID-19 en padres, alumnado y profesorado en ESO y FP. Enseñanza y Aprendizaje de Ingeniería de Computadores, 10, 39-48. https://doi. org/10.30827/digibug.64780 
OMS. (2020). Mental health and psychosocial considerations during COVID-19 outbreak. World Health Organization, January, 1-6.

Reupert, A., y Maybery, D. (2016). What do we know about families where parents have a mental illness? A systematic review. Child and Youth Services, 37(2), 98-111. https://doi.org/10.1080/0145935X.2016.1104037

Salovey, P., Mayer, J. D., Goldman, S. L., Turvey, C., y Palfai, T. P. (1995). Emotional attention, clarity and repair: Exploring emotional intelligence using the Trait Meta-Mood Scale. In J. W. Pennebaker (Ed.), Emotion, disclosure, y health (p. 125-154). American Psychological Association

Sandín, B., Valiente, R. M., García-Escalera, J., y Chorot, P. (2020). Psychological impact of the COVID-19 pandemic: Negative and positive effects in Spanish people during the mandatory national quarantine. Revista de Psicopatologia y Psicologia Clinica, 25(1), 1-22. https://doi.org/10.5944/RPPC.27569

Swann, O. v., Holden, K. A., Turtle, L., Pollock, L., Fairfield, C. J., Drake, T. M., Seth, S., Egan, C., Hardwick, H. E., Halpin, S., Girvan, M., Donohue, C., Pritchard, M., Patel, L. B., Ladhani, S., Sigfrid, L., Sinha, I. P., Olliaro, P. L., Nguyen-Van-Tam, J. S., ... Semple, M. G. (2020). Clinical characteristics of children and young people admitted to hospital with covid-19 in United Kingdom: Prospective multicentre observational cohort study. The BMJ, 370, m3249 https://doi.org/10.1136/bmj.m3249

Tran, T., Hoang, A. D., Nguyen, Y. C., Nguyen, L. C., Ta, N. T., Pham, Q. H., Pham, C. X., Le, Q. A., Dinh, V. H., y Nguyen, T. T. (2020). Toward sustainable learning during school suspension: Socioeconomic, occupational aspirations, and learning behavior of vietnamese students during COVID-19. Sustainability, 12(10), 4195. https:// doi.org/10.3390/sul2104195

Valero, E., Martín, U., y Domínguez, A. (2020). Covid-19 y salud infantil: El confinamiento y su impacto según profesionales de la infancia. Revista Espanola de Salud Publica, 94, 1-7.

Valero, N., Velez, M., Duran, A., y Torres, M. (2020). Afrontamiento del COVID-19: estrés, miedo, ansiedad y depresión. Enfermería Investiga. Investigación, Vinculación, Docencia y Gestión, 5(3), 63-70. file:///C:/Users/Maria Jose/OneDrive/Escritorio/913-110-2157-1-10-20200706.pdf

Zapata, A., Bastida, M., Quiroga, A., Charra, S., y Leiva, J. M. (2013). Evaluación del bienestar psicológico y estrategias de afrontamiento en padres con niños o adolescentes con retraso mental leve. Psiencia Revista Latinoamericana De Ciencia Psicológica, 5(1), 15-23. 